\title{
An insitu borescopic quantitative imaging profiler for the measurement of high concentration sediment velocity
}

\author{
Edwin A. Cowen $\cdot$ Russell D. Dudley \\ Qian Liao • Evan A. Variano • Philip L.-F. Liu
}

Received: 1 July 2009/Revised: 8 December 2009/Accepted: 10 December 2009/Published online: 30 December 2009

(C) The Author(s) 2009. This article is published with open access at Springerlink.com

\begin{abstract}
The design, calibration, and testing of a borescopic quantitative imaging profiler (BQuIP) system, suitable for the insitu measurement of two components of the instantaneous velocity in high sediment concentration flows, are presented. Unlike planar quantitative imaging techniques, BQuIP has a concentration-dependent field of view, requiring detailed calibration. BQuIP is demonstrated in unidirectional sheet flow in an open channel flume with a narrow-graded sand with median diameter $0.25 \mathrm{~mm}$. Acoustic velocity measurements are made in the suspension region above the BQuIP measured region yielding a continuous measurement of velocity and turbulent stress from the immobile bed to just below the free surface. The temporal history at a point reveals the sheet flow sediment velocities to be highly intermittent, and the spectra reveal a broad range of temporal scales close to -5 / 3 in slope for the streamwise velocity component. At its core BQuIP is a quantitative imaging technique giving it
\end{abstract}

E. A. Cowen $(\bowtie)$ · P. L.-F. Liu

DeFrees Hydraulics Laboratory, School of Civil and

Environmental Engineering, Cornell University, Ithaca,

NY 14853-3501, USA

e-mail: eac20@cornell.edu

R. D. Dudley

Wetland Studies and Solutions, Inc., Gainesville,

VA 20155, USA

Q. Liao

Department of Civil Engineering and Mechanics, University of Wisconsin-Milwaukee, Milwaukee, WI 53211, USA

E. A. Variano

Civil and Environmental Engineering,

University of California, Berkeley, Berkeley,

CA 94720-1710, USA significant flexibility in terms of both the spatial and temporal analysis parameters (e.g., interrogation subwindow size and $\Delta t$, the time between images in a pair to be analyzed), allowing it to have tremendous dynamic range in terms of the velocities that can be measured.

\section{Introduction}

A principal challenge to model development for coastal and riverine erosion, morphological, and sediment transport studies is the lack of available data on bedload transport. It has long been recognized (e.g., Bagnold 1966) that bedload is an appreciable, and potentially dominant (e.g., in the swash zone, the region of the beach face that is alternately covered and uncovered by wave runup) component of the total sediment load. While a growing number of acoustic and optical techniques exist for measuring suspended sediment concentration and velocity, the measurement of bedload sediment velocity and concentration remains a significant challenge.

Ribberink and Al-Salem (1995) developed perhaps the first insitu sensor capable of measuring the instantaneous sediment concentration under bedload conditions, the conductivity concentration meter, or CCM. They used the CCM to study the concentration profile in oscillatory sheet flows while Sumer et al. (1996) used the CCM to measure the sediment concentration profile in steady sheet flow conditions, finding a linear concentration dependence with depth in the lower half of the sheet flow region. Notably, Sumer et al. (1996) relied on a Pitot tube with a $0.5-\mathrm{mm}$ orifice to measure the velocity profile within the sheet and were limited to larger diameter sediments (plastic spheres with characteristic diameters of 2.6 and $3.0 \mathrm{~mm}$ ). 
McLean et al. (2001) leveraged the ability of the CCM to continually measure a signal proportional to sediment concentration to develop a 2-CCM approach for the determination of velocity via cross-correlation. They located two CCM sensors $20 \mathrm{~mm}$ apart in the principal flow direction. Using cross-correlation of the two CCM sensor signals, they were able to determine the lag between the arrival of a concentration pattern at the second CCM sensor relative to when the pattern was seen on the first CCM sensor and hence they could estimate the sediment-water mixture's average velocity as the fixed $20 \mathrm{~mm}$ distance divided by the determined temporal lag. The measurement volume of an individual CCM probe is approximately $2 \mathrm{~mm}$ in the flow direction, $1-1.5 \mathrm{~mm}$ in the vertical, and $0.5 \mathrm{~mm}$ in the lateral (McLean et al. 2001) and the determined velocity is the average over the $20 \mathrm{~mm}$ sediment-water mixture path length between the two CCM probes.

The 2-CCM approach to measuring velocity represents a fundamental leap in bedload and high-sediment concentration measurement, in that for the first time, the velocity of the sediment-water mixture can be determined. However, the 2-CCM approach has many limitations:

1. The measurement is an ensemble average as the individual correlations suffer significant noise and ensemble averaging is necessary (McLean et al. 2001) to extract a meaningful estimate of velocity-the ensemble mean velocity.

2. The determined velocity is for the average sedimentwater mixture concentration and not the sediment particle velocity.

3. Since an ensemble average is required, there is an additional uncertainty due to the dynamic position of the bed over the ensemble time, which at the $95 \%$ level is approximately $3 \mathrm{~mm}$ in the work of McLean et al. (2001).

4. The measurement volume is large, and the velocity is calculated over a significant pathlength-on the order of 80 grain diameters for a typical $D_{50}=250 \mu \mathrm{m}$ sand.

5. The downstream CCM in the pair lies in the wake of the upstream CCM sensor biasing the velocity measurement-McLean et al. (2001) estimate the magnitude of this bias to be $5-10 \%$.

6. The fixed spatial separation of the 2-CCM sensors results in a limited range of sediment-water velocities that the 2-CCM approach can resolve (DohmenJanssen and Hanes 2002, 2005).

The final limitation results from the decorrelation of the two CCM signals under lower velocity conditions where the pathlength, $15 \mathrm{~mm}$ in the cases of Dohmen-Janssen and Hanes (2002) and (2005), was too long relative to the correlation length scale of the sediment-fluid mixture. Interestingly, Dohmen-Janssen and Hanes (2005) also had trouble making sediment-water mixture velocity measurements under the highest wave crests, possibly, they argue, due to the thinness of the sheet flow thickness.

Motivated both by the success of the 2-CCM approach for the determination of the sediment-water mixture velocity and its shortcomings, we developed a twodimensional optical approach that allows two components of the instantaneous sediment velocity to be measured robustly. In the remainder of this manuscript we present the measurement approach and results from measurements in uniform sheet flow conditions.

\section{Approach}

The principal challenge to making robust instantaneous measurements of velocity with the 2-CCM approach is its limited degrees of freedom, both in terms of the spatial separation over which the cross-correlation is calculated, and the amount of information contained within the correlated portion of the two signals. Based on our experience with the development of optical measurement techniques (Cowen and Monismith 1997; Cowen et al. 2001; Lara et al. 2002; Liao and Cowen 2005), we leveraged the concept of the 2-CCM approach into an optically based approach. The concept was to replace the 2-CCM sensor pair with a single imaging device, in the tradition of quantitative imaging, capable of capturing sufficient information to allow regions in space to be correlated across images (time).

Our approach is to deliver and collect light locally, within the high sediment concentration region, from a single optical component. Two optical components were investigated for this purpose, a fiberscope and a borescope. The former is a flexible bundle of thousands of extremely small (50-100 $\mu \mathrm{m})$ optical fibers, some of which are used to deliver light and others of which are used to collect scattered light, while the latter is composed of a rigid optical rod lens for the collection of light surrounded by a ring of fibers for the delivery of light. While fiberscopes offer the advantage of a physically flexible optical system, they suffer from inferior image quality relative to similar diameter borescopes and are generally more expensive. Given our intention of mounting the optical components on a 1-D computer-controlled positioning stage and the improved optical quality at a given diameter, the borescope was selected as our optical delivery and collection device. Working with Volpi USA (Auburn, NY), we balanced minimizing diameter, and hence intrusiveness, while maximizing image quality and selected a 2.7-mm OD Volpi Intrascope with a $90^{\circ}$ prism.

Our image collection tests of light scattered at the distal tip of the borescope submerged in a sand bed revealed that 
excellent image quality could be attained if sufficient light was delivered. Given the relatively high velocity of sheet flows, the target flow for our measurement system, we need to deliver light over a relatively short time scale to avoid motion-induced image blur, and under these conditions, the amount of available light becomes the limiting factor in the quality (dynamic range) of the images. For cost and deployment flexibility, our goal was to work with white light sources and our needs suggested a strobed illumination source. We selected a Perkins Elmer LS1130-4 FlashPac fitted with a FX-1163 Xenon flashlamp with a maximum pulse rate of $1 \mathrm{kHz}$ and a maximum average power of $20 \mathrm{~W}$. Given our plan to use a particle image pattern matching or correlation-based approach and the intended optical magnifications, pulse separations of $0.5 \mathrm{~ms}$ are insufficient, so we elected a frame straddling approach based on the use of two FlashPacs, similar to a dual Nd:YAG approach for illumination in particle image velocimetry (PIV).

Despite the use of the high-powered pulsed white light source limited light availability was still an issue and to increase the optical efficiency of our image collection system, a 12-bit digital CCD camera, a Uniq Vision, Inc. UP-680-CL-12B (494 × 659 pixel array, $9.9 \mu \mathrm{m} \times 9.9 \mu \mathrm{m}$ pixels, maximum full-frame frame rate $60 \mathrm{~Hz}$ ), was selected. This is an interline transfer camera allowing frame transfers to occur in less than $20 \mu \mathrm{s}$. The Intrascope is mounted via a $\mathrm{C}$-mount coupler to the camera yielding a circular image at the pixel array with approximate diameter of 340 pixels. The FlashPacs are connected to the Intrascope with a custom flexible lightguide (Volpi USA) allowing the two FlashPacs to each illuminate the fiber illumination ring of the Intrascope. The FlashPacs are triggered by TTL input signals allowing arbitrary time between image pair illuminations, $\Delta t$, ranging between the camera frame rate down to the interline transfer time.

While in theory, 2-D cross correlation could be used to analyze the image pairs, this would bias the measurement of velocity toward sand grains that imaged brightly (e.g., whites) relative to the darker sand grains. To utilize all of the captured image intensity information, the minimum quadratic difference (MQD, Gui and Merzkirch (1996, 2000)), calculated as in Eq. 1, is employed.

$D(m, n)=\sum_{i=-R_{y}}^{R_{y}} \sum_{j=-R_{x}}^{R_{x}}[F(i, j)-G(m+i, n+j)]^{2}$

where $\pm R_{x}$ and $\pm R_{y}$ are the horizontal and vertical extent of the calculation region, $F$ and $G$ are the interrogation subwindows in the first and second image of an image pair, respectively, and $D$ is the MQD. Note that this is a direct calculation, and no assumption of periodicity is invoked.

While a 3-point (1-D) Gaussian subpixel correlation peak displacement estimator has been demonstrated to be optimal for particle image diameters of order 1 pixel (Cowen and Monismith 1997; Westerweel 1993), we are aware of no similar analysis for determining to subpixel location the MQD minimum in $D$. In an effort to determine the optimum subpixel minimum estimator, we computationally displaced a set of 200 images collected in our sediment flume under sheet flow conditions (see Sect. 3 for details) at an elevation of $z=1.250 \mathrm{~mm}$ (see Sect. 3 for coordinate system) with a step of 0.02 pixels over 1 pixel (i.e., 51 steps) using a 2-D spline interpolator (results were found to differ by less than $6 \%$ when a bilinear interpolator was used) to generate 200 image pairs of known subpixel displacement. The MQD algorithm was applied to determine the integer pixel location of the minimum, and a 1-D Gaussian and 2-D centroid calculated on a $3 \times 3$ kernel were used to estimate the subpixel displacement of the minimum. The mean and RMS errors, relative to the applied displacements, are shown in Figs. 1 and 2, respectively.

The centroid has an advantage in mean error while suffering greater RMS errors relative to a Gaussian estimator. Given these competing trends, the average result of the two estimators was also calculated and is shown in Figs. 1 and 2.

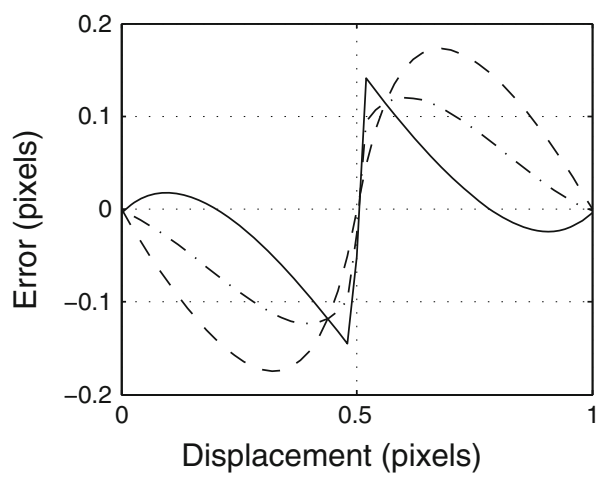

Fig. 1 Mean error as a function of subpixel displacement for: solid line $3 \times 3$ centroid, dashed line 3 point Gaussian, and dash-dotted line the average result of the centroid and Gaussian estimate

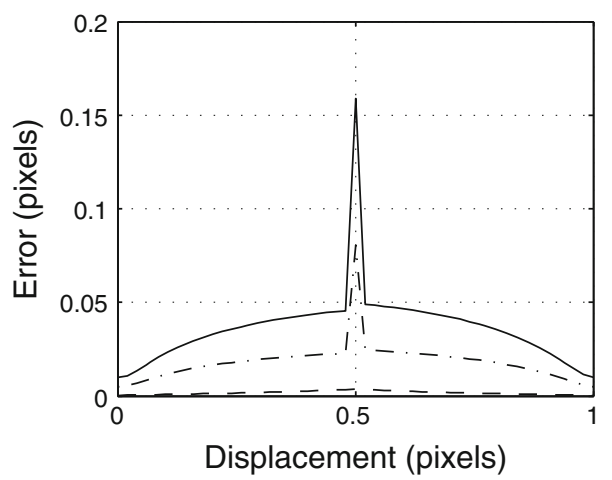

Fig. 2 RMS error as a function of subpixel displacement for: solid line $3 \times 3$ centroid, dashed line 3 point Gaussian, and dash-dotted line the average result of the centroid and Gaussian estimate 
The total averaged-over-one-pixel error for the three schemes, taking the absolute value of the mean error, is 0.08, 0.11, and 0.09, respectively (centroid, Gaussian, average), suggesting that any of the three subpixel fit schemes is a reasonable choice and should be selected based on the trade-off for a particular experiment on controlling random error vs. bias errors. We have elected to use the $3 \times 3$ centroid for the remainder of this article as it has the lowest total error while minimizing the bias error.

The use of an optical approach presents a new challenge relative to the 2-CCM approach and more traditional planar quantitative imaging techniques (e.g., Cowen and Monismith 1997), the measurement volume size, and hence the calibration, is a function of sediment concentration.

\section{Experiment}

In order to test our approach and develop a method for handling the calibration of the measurement, an experiment was carried out in the DeFrees Hydraulics Laboratory in the School of Civil \& Environmental Engineering at Cornell University. A recirculating-type tilting sediment flume with a $9 \mathrm{~m}$ long, $0.60 \mathrm{~m}$ wide, and $0.45 \mathrm{~m}$ deep Plexiglas test section supported by a steel frame and a plywood subbed was used. The bed slope, $S=0.0035$, was set to achieve uniform flow at the specific test conditions described below. The inlet section was fitted with a custom built honeycomb-type grid constructed from $0.60 \mathrm{~m}$ long sections of $2^{\prime \prime}$ ID PVC pipe, oriented vertically in the inlet with the exit plane of the honeycomb located below the flume bed plane. A variable speed centrifugal pump was used to recirculate the flow. An inline polyethylene storage tank, approximate volume $2 \mathrm{~m}^{3}$, was located between the flume outlet and the pump in order to minimize fluctuations in pump efficiency.

The camera/borescope system was mounted $6.3 \mathrm{~m}$ downstream from the beginning of the test section at the lateral midpoint of the facility on a 1-D computer-

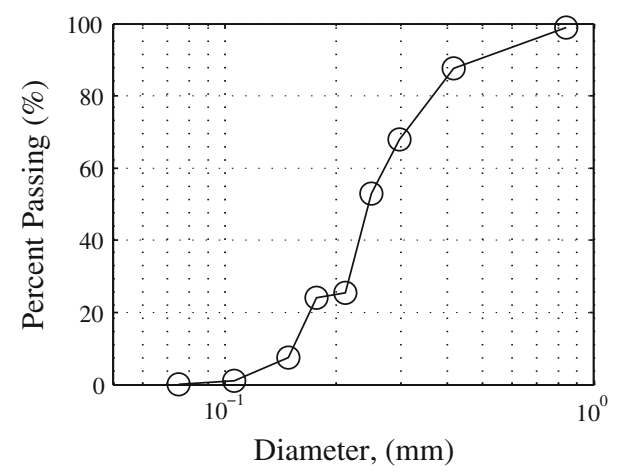

Fig. 3 Sieve analysis of the sand used controlled stage with $300 \mathrm{~mm}$ of travel (Velmex UniSlide MA1500 series driven by a Animatics SM 1720D85C SmartMotor). The flume was filled to an average depth of approximately $6 \mathrm{~cm}$ with a commercial sandbox sand, sieved of debris and gravel, and rinsed repeatedly to remove the fines. The results of a sieve analysis carried out on the rinsed sand are shown in Fig. 3 demonstrating the sand to be narrowly graded with a median diameter $\left(D_{50}\right)$ of $0.25 \mathrm{~mm}$. The Flashpacs were mounted on the flume frame and attached to the borescope with the custom flexible lightguide. The borescope passed through adjustable Swagelok tube fittings at both the top and bottom interfaces of the flume bed, ensuring a water- and sandtight seal. The complete system, dubbed a Borescopic Quantitative Imaging Profiler (BQuIP), is shown in Fig. 4.

Images were acquired using Boulder Image's (Louisville, CO) VisionNow software to control a 64-bit Camera Link capture board. Timing and control signals were generated by MathWorks MATLAB and a National Instruments PCMCIA DAQCard-6715.

We define the experimental coordinate system such that $x$ is in the direction of flow, parallel to the bed, $z$ is defined vertically normal to the bed, positive upwards, and $y$ is in the transverse direction as defined by the right-hand-rule with $y=0$ defined at the lateral midpoint of the flume. The optical axis of the borescope is aligned with the $y$ coordinate direction, which is orthogonal to the principal flow direction, $x$. We define $\xi$ as the optical axis of the borescope and hence it is collinear with $y$, increasing with distance from the borescope. The origin $\xi=0$ is defined as the intersection of the outer diameter of the borescope with the optical axis.

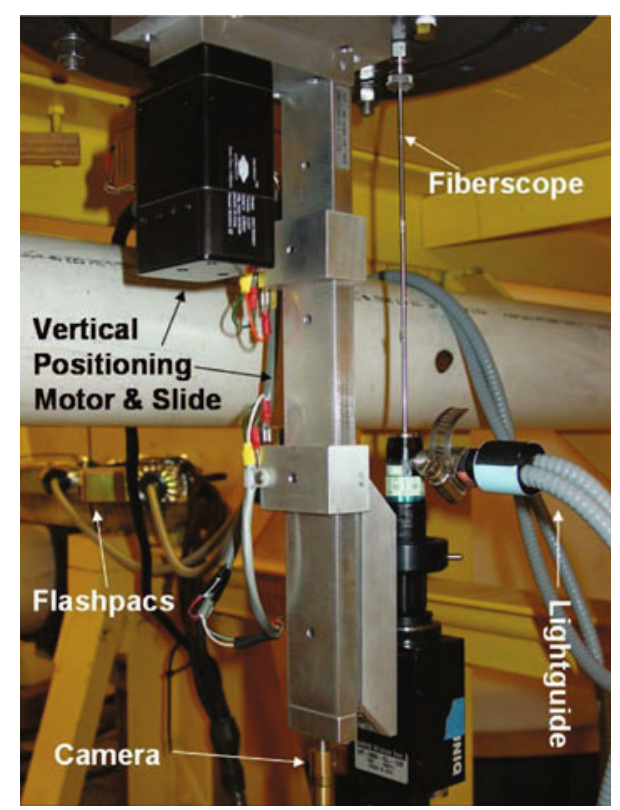

Fig. 4 The BQuIP system mounted beneath the sediment flume 
Water velocities were measured with a Nortek Vectrino+ acoustic Doppler velocimeter (ADV) mounted vertically from above directly over the BQuIP. The ADV was operated in high power mode and sampled at $50 \mathrm{~Hz}$ at a velocity range of $1.00 \mathrm{~m} / \mathrm{s}$ and transmit length and sampling volume lengths of 2.4 and $9.1 \mathrm{~mm}$, respectively. All ADV sample records were collected over $10 \mathrm{~min}(30,000$ samples). The BQuIP was retracted well below the mobile part of the bed while ADV measurements were carried out.

The flume was operated at a water depth $h=14.8 \mathrm{~mm}$ and a depth-averaged flow speed of $U_{o}=0.99 \mathrm{~m} / \mathrm{s}$ (Froude number $\mathrm{Fr}=0.89$ ), sufficient to establish sheet flow conditions. The Shields parameter, the non-dimensional bed stress, defined as

$\Theta=\frac{u_{*}^{2}}{g D_{50}(s-1)}$

where $u_{*}$, the friction velocity, is defined as in Eq. 5 and $s=\rho_{\text {sed }} / \rho$, the ratio of the sediment density to the water density, was $\Theta=1.0$. The value of $\Theta=1.0$ was specifically chosen to ensure that a steady plane sheet flow was generated. The flume was operated for approximately $2 \mathrm{~h}$ until a steady state bed was observed within $\pm 1 \mathrm{~m}$ from the measurement location prior to the commencement of BQuIP measurements. BQuIP measurements were initiated at a vertical position below the point where the bed was mobile. Image pairs were collected at $10 \mathrm{~Hz}$ for $10 \mathrm{~min}$ yielding typically 5,999 image pairs per set (the first image in each set was corrupted hence the loss of one image pair). The sets were collected every $0.250 \mathrm{~mm}$ in the vertical over a $6 \mathrm{~mm}$ range, covering from the region where the sediment was clearly immobile to the point where the BQuIP measurement volume crossed through and out of the sheet flow region.

Due to light limitations, the dynamic range of the images was low, typically about 45 counts, and the images suffered from high frequency random noise. To enhance data quality, the images were preprocessed. A pixelwise adaptive two-dimensional Wiener filter based on statistics estimated from the local $3 \times 3$ neighborhood of the grayscale image is first applied. This result is then twodimensional median filtered, also on a $3 \times 3$ neighborhood. This combination proved to be an effective low-pass filter allowing the sharp sediment grain boundaries to be preserved while removing the high frequency random noise. As the illumination intensity varied spatially across the images, the images were then histogram equalized.

The image pairs were analyzed using four subwindows, each 105 pixels in the $x$ direction and 48 pixels in the $z$ direction. The image path in the borescope contained pixels that did not respond to light, probably due to dust in the optical path. The subwindows were located to avoid these regions, as shown in Fig. 5. The upper-left corner of the

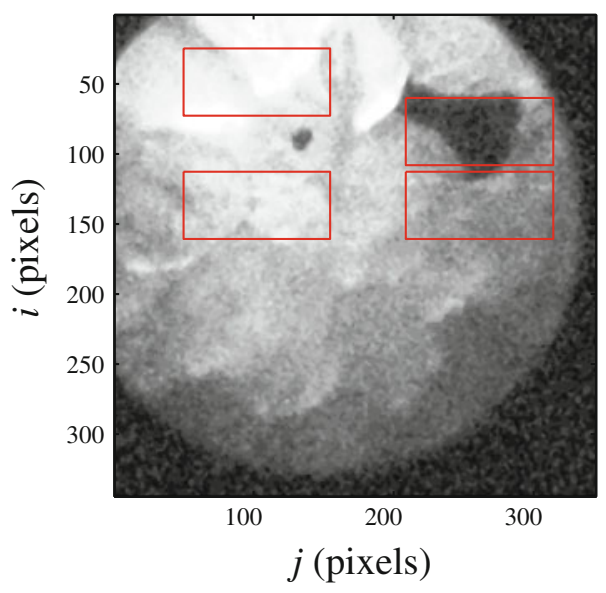

Fig. 5 Typical preprocessed BQuIP image showing interrogation subwindows. Note the dark spot located around $(90,120)$ is a constant in the image set while the dark larger grain-like image located in the top-right subwindow is a sediment grain image

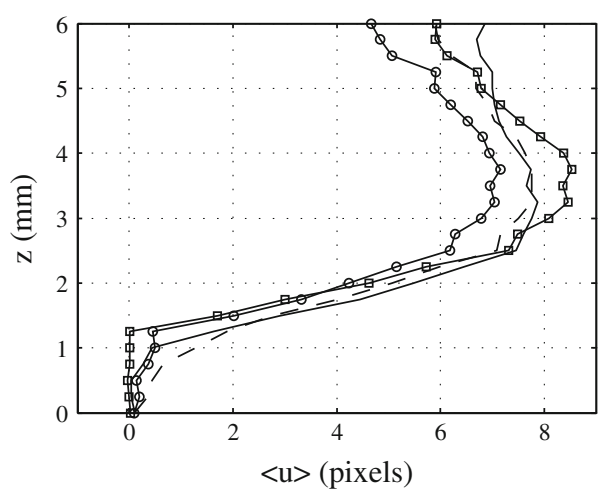

Fig. 6 Mean MQD calculated displacement profiles from the four subwindows: upper-left solid line, upper-right dashed line, lower-left $\square$, lower-right $-\circ$

four subwindows were located at the indices: $(25,50)$, $(113,50),(60,209),(113,209)$, where the indices are $(i, j)$. Calculated displacements were filtered using an adaptive Gaussian window (Cowen and Monismith 1997) on the temporal series at each subwindow location with $4.5 \%$ of the displacements being removed as spurious. For the calculation of spectra, the spurious data points were replaced by linear interpolation in time; a reasonable choice given the low spurious vector fraction (Poelma et al. 2006). For all other results calculated from the displacements, no interpolation was used.

Figure 6 shows the resultant mean displacement profiles determined at each of the four subwindows. The effect of decreasing sediment concentration with distance above the immobile bed is clear. Initially, the displacement gradients are so large that despite increases in the field of view (FOV), the displacement profile behaves as expected, increasing rapidly with distance. However, as the BQuIP 
rises further and the sediment concentration continues to drop, the displacement gradient slows and changes sign, clearly demonstrating the need for calibration.

\section{Calibration}

The lower the sediment concentration, the further, on average, light may travel from the illumination window at the distal tip of the borescope into the sediment-fluid mixture, be scattered by a sediment particle, and return to the image collection window of the borescope, without image degradation due to multi-path light scattering effects. Figure 7 shows the distal tip of the borescope along with depictions of the illumination region and the FOV, or image collection region. The FOV increases in each dimension linearly with distance from the principal axis of the borescope, while the illumination intensity decays approximately as the square of this distance. Note since light emits on the distal tip side of the image collection window there is a region $\xi<2 \mathrm{~mm}$ that is not directly illuminated, hence light intensity increases with increasing $\xi$ to about $\xi=2 \mathrm{~mm}$ and then decays for $\xi>2 \mathrm{~mm}$, as seen in Fig. 8.

Based on a hypothesis that, at a given sediment concentration, collected light is statistically dominated by light scattered from a particular distance to the image collection

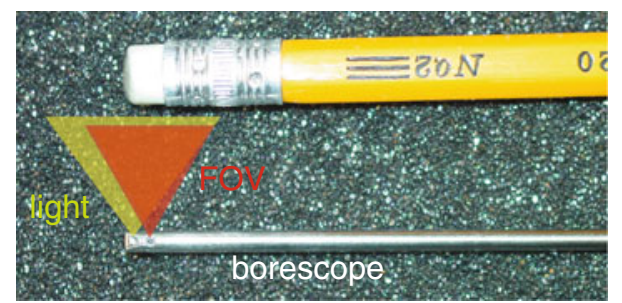

Fig. 7 Distal end of $2.7 \mathrm{~mm}$ OD borescope equipped with $90^{\circ}$ prism for illuminating and viewing orthogonal to the principal borescope axis

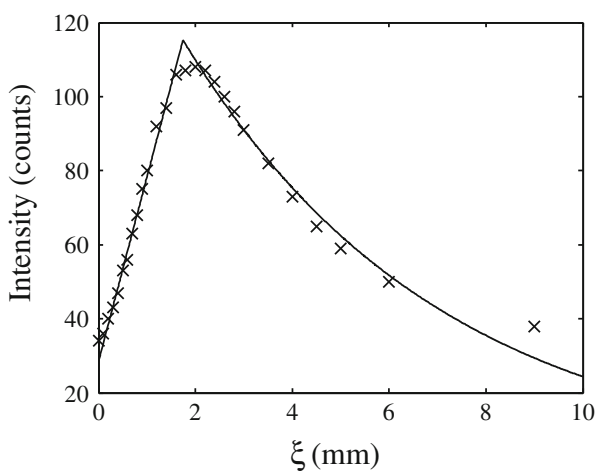

Fig. 8 Median maximum intensity profile from calibration image set along with rising and falling limb curve fits window, we developed a calibration approach that utilizes images of natural sands on a planar sheet moving at a constant velocity relative to the borescope at a series of fixed distances. Multiple layers of the sand used in this experiment were glued to a rigid planar sheet and mounted to a 1-D computer-controlled stage. The borescope was mounted parallel to the planar sheet. The FOV was aimed with its optical axis, $\xi$, orthogonal to the planar sediment sheet. The first 1-D stage allowed the distance of the planar sediment sheet to be set relative to the image collection window on the borescope, e.g., the value of $\xi$ to be adjusted. The second 1-D stage allowed the borescope to be driven at a constant velocity of $0.1 \mathrm{~mm} / \mathrm{s}$ in a plane parallel to the planar sediment sheet (i.e., orthogonal to $\xi$ ).

Image sets were collected at 27 distances (values of $\xi$ ) from the image collection window at $10 \mathrm{~Hz}$ for $200 \mathrm{~s}(2000$ images per set). A square region, $344 \times 344$, containing the FOV was analyzed for five metrics: the median, the 25 th and 75th percentile value, and the 10th smallest and largest values (termed the minimum and maximum values, respectively). The median of these five metrics was calculated across the image set at each distance demonstrating the expected intensity variation based on the illumination-FOV geometry with the maximum metric showing the greatest dynamic range (see Dudley 2007, for plot and a photograph of the moving calibration system). The maximum metric, along with a curve fit (linear on the rising limb, exponential on the falling limb, $R^{2} 0.993$ and 0.974 , respectively, see Dudley (2007) for details), is shown in Fig. 8.

The first image in 600 image pairs collected in the experiment, described in Sect. 3, was processed identically, and the maximum statistic and the same functional form curve fits $\left(R^{2} 0.981\right.$ and 0.892 , respectively, see Dudley (2007) for details) are plotted in Fig. 9 as a function of $z$. In the calibration phase, we define $z=0$ to be at the initially selected lowest vertical elevation measured, which is established such that the sediment velocity is seen clearly

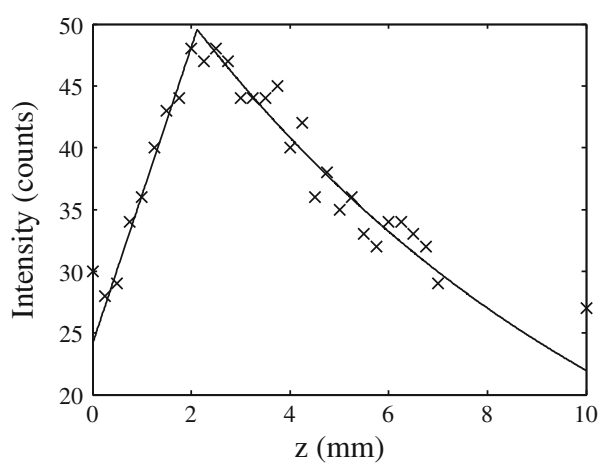

Fig. 9 Median maximum intensity profile from insitu sheet flow experiment image sets along with rising and falling limb curve fits 


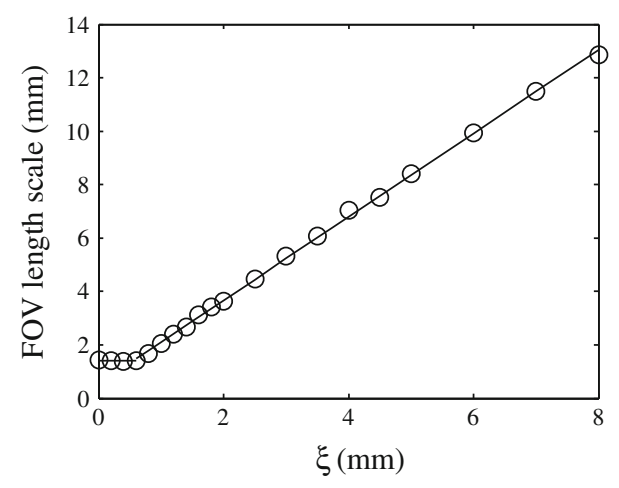

Fig. 10 Spatial calibration of BQuIP system as a function of image plane distance from image collection window

to be zero by looking at the BQuIP images. There is a striking similarity in the general characteristics of Figs. 8 and 9 that was anticipated due to the essentially monotonic drop in sediment concentration with distance above the immobile bed and the associated increase in the effective FOV of the borescope.

The peak intensity is used to normalize each data set, and the insitu set is then scaled to the calibration curve fits based on either the rising limb or falling limb side of the peak maximum intensity location.

The representative distance $\xi$ from the FOV to the image collection window is converted to a spatial calibration using standard quantitative imaging approaches, namely, the interrogation of an image of a scale or object of known physical dimension collected at the appropriate distance from the image plane. For standard planar techniques, this calibration step is done only at one object plane, but the BQuIP requires the calibration to be determined at all anticipated distances $\xi$. In the present case, a wire of known diameter was attached to a dark background that was mounted on a 1-D computer-controlled stage. The wire was moved a series of distances, $\xi$, from the image collection window of the borescope, and the wire's image and its known diameter were used to calibrate the system as a function of $\xi$. The resultant calibration plot is shown in Fig. 10. The figure demonstrates that there are two optical regions as a function of distance from the image collection window: the constant $\mathrm{FOV}$ region for $\xi<0.6 \mathrm{~mm}$ where the FOV remains fixed at $1.40 \mathrm{~mm}$ (magnification factor of 2.42 , likely the diffraction limit of the optical system), and the standard geometric optics region $(\xi>0.6 \mathrm{~mm})$ where the FOV increases at linear rate of $57.4^{\circ}$.

Our expectation was that a calibration metric of maximum intensity would work well for the highest sediment concentration measurements but that as the concentra metric of intensity variance would be more robust to this bias and determined a calibration based on the median image intensity variance in an analogous manner to that

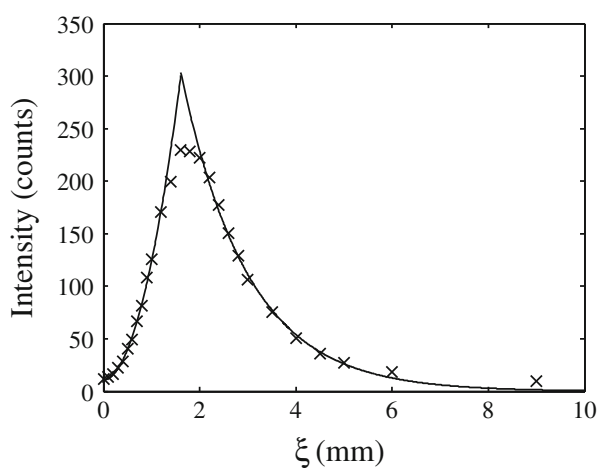

Fig. 11 Median intensity variance profile from calibration image set along with rising and falling limb curve fits

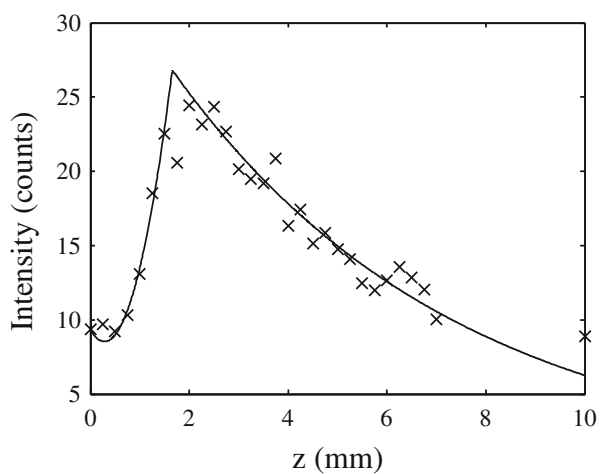

Fig. 12 Median intensity variance profile from insitu sheet flow experiment image sets along with rising and falling limb curve fits

just described for intensity itself. The resulting profiles of variance as a function of $\xi$ for the calibration and $z$ for the insitu sheet flow data sets, along with curve fits to the profiles, are shown in Figs. 11 and 12, respectively. In this case, the rising limb curve fit function is quadratic while the falling limb remains exponential. The $R^{2}$ values for the calibration image set are 0.998 and 0.996 , respectively, while for the insitu image set, they are 0.990 and 0.929 , respectively (see Dudley 2007, for details).

Applying the two calibration result approaches to the mean displacements, along with the imposed $\Delta t=$ $0.100 \mathrm{~ms}$, yields the sediment velocity profiles shown in Fig. 13, where the mean has been taken across the four subwindows. We have now redefined $z=0$ to be the point of incipient motion in the sediment column, i.e., for $z>0$, the sediment bed is, on average, moving, and for $z<0$ it is stationary. We note that based on the original definition of $z=0$, we have offset the profile by $1.0 \mathrm{~mm}$, i.e., if the new origin was applied to Fig. 12 and earlier, they would begin at $z=-1.0 \mathrm{~mm}$ or, the originally labeled $z=1.0-\mathrm{mm}$ grid line is the new $z=0$ grid line. Interestingly, the results do not bear out our assumption that the maximum intensity metric would underestimate the intensity variance-based metric, and we remain uncertain as to the reason. The two 


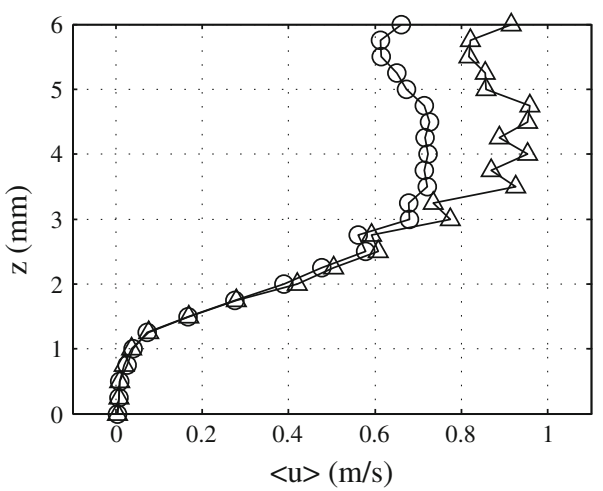

Fig. 13 Mean BQuIP measured velocity profile: intensity variance method circle, intensity maximum method triangle

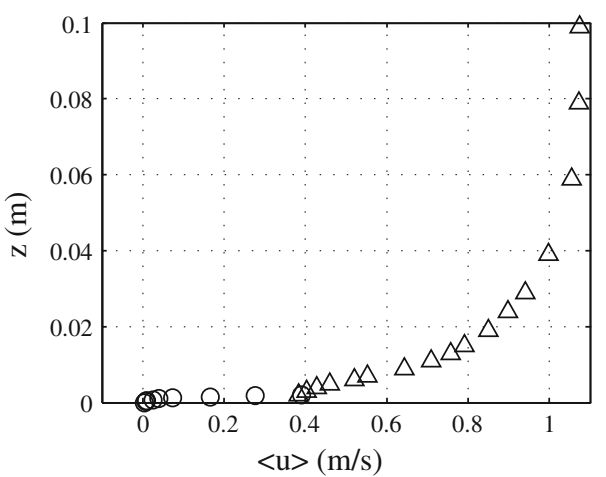

Fig. 14 Total mean velocity profile: $\mathrm{BQuIP}$ circle, $\mathrm{ADV}$ triangle

calibration approaches produce identical results below $z=2.0 \mathrm{~mm}$ and are nearly in agreement up to $z=2.5 \mathrm{~mm}$.

Looking at these results in the context of the ADVmeasured velocity profile reveals that the borescope and ADV measurement volumes are at the same vertical point in the flow when the borescope is positioned $2.0 \mathrm{~mm}$ above the immobile bed and the ADV measurement volume is reported as $3.0 \mathrm{~mm}$ above the immobile bed. Combining the two profiles yields the plots which are shown in Figs. 14 and 15. Note that either calibration method appears to work well in the high concentration region of the flow below the region where the ADV measurements are valid. We prefer the intensity variance method at this point as we believe it to be more robust, particularly as the concentrations get lower, as it is a metric incorporating all spatial data while the median maximum intensity is not weighted by the spatial data.

Given the more complex nature of calibrating the BQuiP, we summarize the procedure as follows:

1. Determine the variation of the FOV with $\xi$, the distance to the object plane (i.e., produce Fig. 10).

2. Glue multiple layers of sand under study to a rigid planar substrate and mount on a 1-D traverse.

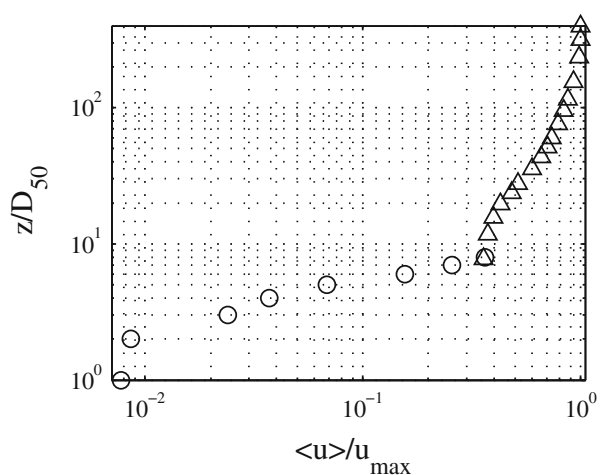

Fig. 15 Total mean velocity profile: BQuIP circle, ADV triangle

3. Mount BQuIP with $\xi$ orthogonal to rigid planar substrate at a fixed distance $\xi$ and collect images of the sand pattern traversing by the BQuIP at a constant speed.

4. Analyze collected image sequence for metrics of interest-in our case, the median intensity variance.

5. Repeat steps 3 and 4 over the range of $\xi$ of interest.

6. Curve fit the metric of interest (e.g., median intensity variance) as a function of $\xi$ (i.e., produce Fig. 11).

7. Carry out actual experiment making measurements as a function of $z$ with $z=0$ set at an arbitrary depth, where the sediment velocity clearly is zero.

8. Curve fit the metric of interest (e.g., median intensity variance) from the experimental image set as a function of $z$ (i.e., produce Fig. 12).

9. Normalize the calibration curve fits and the insitu experimental curve fits by the respective peak in the metric (e.g., value at approximately $\xi=2 \mathrm{~mm}$ and $z=2 \mathrm{~mm}$ in our case, respectively).

10. Estimate the equivalent value of $\xi$ for the given $z$ from the normalized curve fits.

11. Use the results of step 1 to get the spatial calibration based on the step $10 \xi$ estimate.

12. Adjust the position of $z=0$ to be coincident with the vertical elevation of incipient sediment motion.

We emphasize that our calibration procedure has only been shown to be effective for a narrowly graded noncohesive sand. The procedure will need to be verified for other sediment types and diameter distributions.

The calibration procedure does not allow us to directly assess the actual measurement volume, as we have no direct measure of the maximum extent the BQuIP can see into the flow (maximum $\xi$ ). Applying our final calibration to the entire measured $z$ range of the insitu experiments yields an estimate of the diameter of the FOV as a function of $z$ as shown in Fig. 16. Given the principle measurement region of interest lies in the range $0 \leq z \leq 2.5 \mathrm{~mm}$, we see the measurement volume's characteristic length scale 


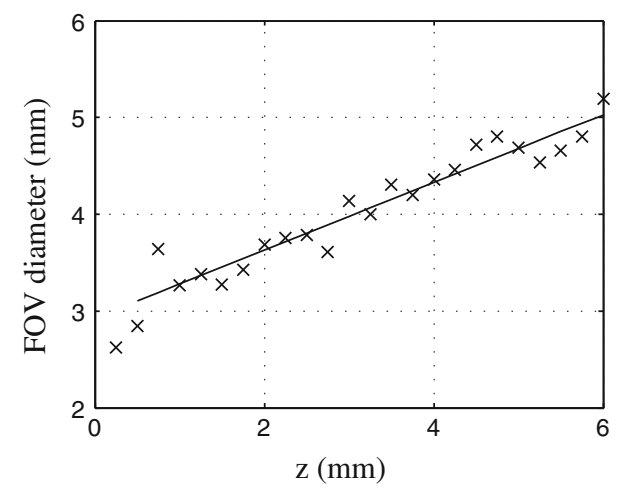

Fig. 16 FOV diameter as a function of $z$ based on calibration

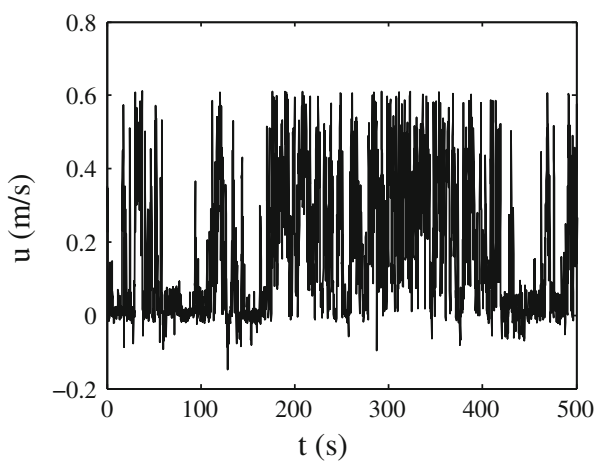

Fig. $17 u$ velocity record at upper-left subwindow at $z=1.25 \mathrm{~mm}$. Note mean velocity at this location is $0.13 \mathrm{~m} / \mathrm{s}$

ranges from 2.6 to $3.7 \mathrm{~mm}$; small relative to the $2-\mathrm{CCM}$ probe technique yet large relative to the $D_{50}$. We note the near-linear variation in the measurement volume size is consistent with the Sumer et al. (1996) finding of a linear variation with depth in sediment concentration in the sheet flow regime.

\section{Discussion}

Given our instantaneous calibrated BQuIP measurements of sediment velocity, we are able to calculate various turbulent quantities within the sheet flow regime. Before looking at these quantities, it is informative to look at the instantaneous velocity time history. Figure 17 shows $500 \mathrm{~s}$ of the velocity record from the upper-left subwindow at $z=1.25 \mathrm{~mm}$, where the mean velocity is $0.13 \mathrm{~m} / \mathrm{s}$. Note the strong intermittency of the flow at all time scales. Figure 18 shows the ensemble averaged spectra of the streamwise $(u)$ and vertical $(w)$ velocity at the same location, where the temporal record has been broken into four ensembles to allow the ensemble averaging. The spectra demonstrate a strong anisotropy in the fluctuations, at all frequencies as well as the strong energy cascade in the streamwise fluctuations. This is true at all elevations within

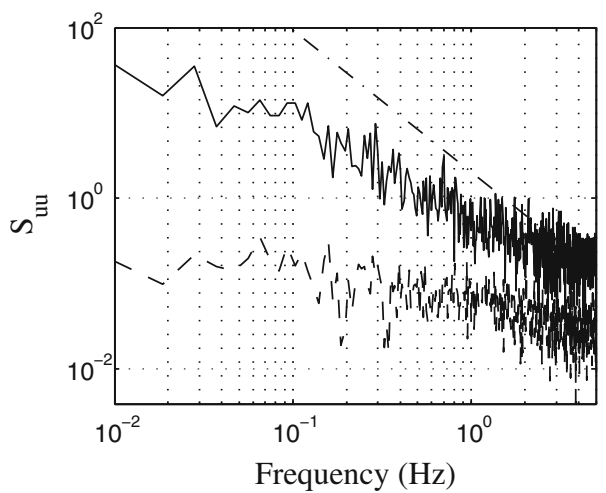

Fig. 18 Spectra of each component of the velocity at upper-left subwindow at $z=1.25 \mathrm{~mm}$ : $S_{u \text { u }}$ solid line, $S_{w w}$ dashed line, $-5 / 3$ sloped dash-dotted line

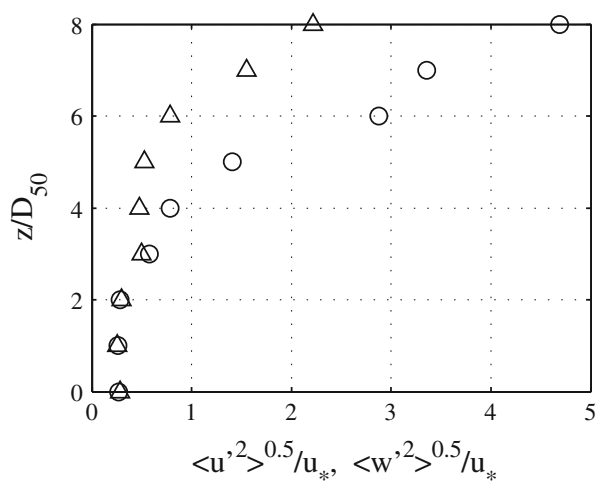

Fig. 19 Turbulent intensities as measured by BQuIP non-dimensionalized by $u_{*}$ : circles $\sqrt{\overline{u^{\prime 2}}}$, triangles $\sqrt{\overline{w^{\prime 2}}}$

the borescope-measured region, once appreciable sediment motion sets in, around $z=1.00 \mathrm{~mm}$.

The turbulent intensities, defined based on Reynolds decomposition as:

$\sqrt{\overline{u^{\prime 2}}}$ where $u^{\prime}=u-\bar{u}$

and $\overline{()}$ indicates the temporal average, are shown in Fig. 19. Data is shown from the BQuIP through an elevation of $8 D_{50}(2 \mathrm{~mm})$ above the immobile bed, consistent with the mean flow data shown in Figs. 14 and 15. The strong anisotropy in the fluctuations is apparent.

A fundamental quantity of interest in the study of sediment laden flows is the friction velocity. The standard approach to its calculation in the lab is to use a momentum balance, which leads to

$u_{*}=\sqrt{g R S}$

where $g$ is the acceleration of gravity and $R$ is the hydraulic radius, defined as the ratio of the cross-sectional flow area divided by the wetted perimeter. This is the total momentum balance, which includes the effects of the bed stress and the side wall stress. Vanoni and Brooks (1957), 
in their Appendix A, provide a method to isolate the bed stress which is handled by solving for the effective hydraulic radius of the bed, $R_{\mathrm{b}}$, hence

$u_{*}=\sqrt{g R_{\mathrm{b}} S}$.

Equation 5 was applied finding $u *=65 \mathrm{~mm} / \mathrm{s}$ or $\tau_{b}=\rho u_{*}^{2}=4.2 \mathrm{~N}$, where $\rho$ is the density of water. The BQuIP and ADV stress are calculated as $\tau=-\rho_{\text {sed }} \overline{u^{\prime} w^{\prime}}$, where $\rho_{\text {sed }}$ is the density of the sediment, taken as $2,600 \mathrm{~kg} / \mathrm{m}^{3}$. While the choice $\rho_{\text {sed }}$ seems fairly clear for the BQuIP, we feel for the same reasons, namely, both the BQuIP and the ADV are measuring reflected light and sound, respectively, from sediment particles, the choice is clear for the ADV as well. To test our stress calculation from the ADV, we plotted the stress based on the measured $\overline{u^{\prime} w^{\prime}}$ and both $\rho$ and $\rho_{\text {sed }}$ as shown in Fig. 20, where the stress based on $\rho$ would be the case if the ADV scatterers are passive (specific gravity 1 ).

Figure 20 clearly demonstrates that the normalized stress profile goes to approximately 1 in the limit of the bed, as it should, only when the stress is calculated based on the appropriate density of the scatterer, in this case the sediment density. We note that the linear extrapolation of the stress to the bed yields $-\rho_{\text {sed }} \overline{u^{\prime} w^{\prime}} / \tau_{b}=1.2$. We hypothesize that the measurement volume scatterers are a mix of the narrowly graded commercial sand and residual near neutrally buoyant scatterers, such as our standard hollow glass spheres used for PIV and ADV seed in the facility (Sphericel, median specific gravity 1.1). Well away from the sediment bed, there is sufficient seed to maintain excellent ADV signal to noise ratio, and this is clearly due to passive tracers suspended in the water column. Near the bed, while sediment is the dominant source of scatters, the passive scatterers still play a role and suggest the need to determine the effective scatterer density as a weighted average of the measurement volume acoustic scatterers. This may be an important area of research for the use of acoustic measurements in particle laden flows.

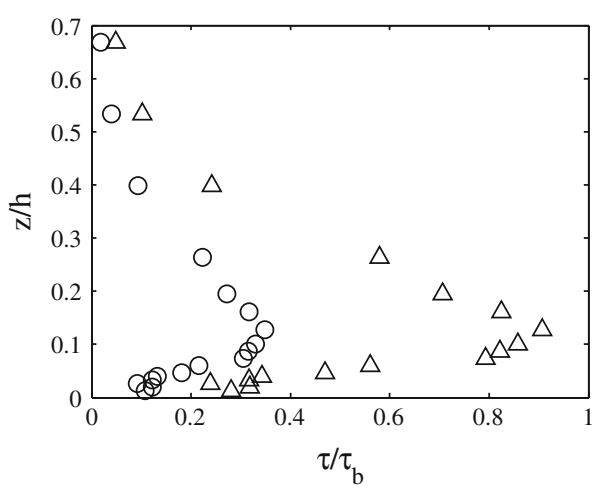

Fig. 20 ADV-measured stress profile normalized by the momentum balance calculated bed stress, $\tau_{b}$ : triangles $\tau=-\rho_{\text {sed }} \overline{u^{\prime} w^{\prime}}$, circles $\tau=-\rho \overline{u^{\prime} w^{\prime}}$
The total stress profile, combining both the BQuIP and ADV measurements, is shown in Fig. 21. Notably, the BQuIP appears to be overestimating the stress at the top of the sheet by about a factor of 2 . The reasons for this are not entirely clear, but one possibility is that motions parallel to the optical axis of the imaging system lead to magnification changes that are correlated in both $u$ and $w$ and may appear as a source of correlated noise in the stress calculation. This will be the subject of future research.

In an effort to further validate the BQuIP data, a bootstrap uncertainty interval (Efron and Tibshirani 1993) at the $95 \%$ confidence level was determined for the random component of the uncertainty for the mean, turbulent intensities, and the turbulent stress, with typical values presented in Table 1. The percentages shown are based on the largest bootstrap reported interval in the $1 \leq z \leq 2 \mathrm{~mm}$ range when normalized by the reported value of the statistic. The largest values in this range are seen to be small and typical values are approximately $2 \%$ of the measured value, demonstrating that the BQuIP is not subject to strong random errors. The determination of a bias error is more problematic. We lack any basis to estimate how the calibration is affected by the instantaneous and mean behavior of particles within the ill-defined and likely dynamic measurement volume.

McLean et al. (2001) found that the downstream CCM in the 2-CCM probe lies in the wake of the upstream CCM sensor biasing the velocity measurement. Given that the BQuIP is intrusive, it is important to try to get a sense for its effect on the flow. Based on the measured velocities the

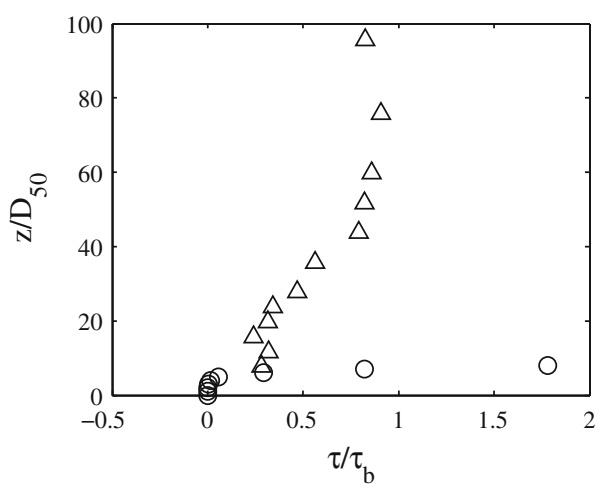

Fig. 21 Total stress profile normalized by the momentum balance calculated bed stress, $\tau_{b}$ : ADV triangle, BQuIP circle

Table 1 Bootstrap determined $95 \%$ confidence interval limits on random error component of turbulence metrics

\begin{tabular}{llllll}
\hline Statistic & $\bar{u}$ & $\bar{w}$ & $\sqrt{\overline{u^{\prime 2}}}$ & $\sqrt{\overline{w^{\prime 2}}}$ & $\overline{u^{\prime} w^{\prime}}$ \\
\hline Low limit (\%) & -3.2 & -3.8 & -2.1 & -2.1 & -4.9 \\
High limit (\%) & +2.2 & +3.8 & +2.0 & +2.4 & +5.2 \\
\hline
\end{tabular}


Reynolds number $(R e)$ based on the borescope cylinder diameter varies from 0 to a maximum in the sheet flow regime of about 1,000. At all but the smallest $R e$, a Karman vortex street is expected with a characteristic shedding frequency. However, this effect would be downstream in the wake. We are imaging from the transverse side of the borescope where the flow is still attached and the boundary layer is very thin (considerably less than one diameter for all but the lowest $R e$ ). Given the insitu measurements returned a minimum distance to object plane $\xi=1.5 \mathrm{~mm}$ or $\xi /$ $D=0.56$ before particle motion set in, the measurement region is well outside of the boundary layer around the probe tip. The spectra (Fig. 18) further confirm this to the extent that the characteristic frequency of the Karman vortex street does not show up. Hence, we conclude that the BQuIP diameter is sufficiently small to have essentially no effect on the flow it is measuring for a narrowly graded sand at this $D_{50}$.

A final point of interest in sediment laden flows is the effect of the suspended sediment on the van Karman constant, $\kappa$. Given that sheet flows provide roughness values greater than expected based on $D_{50}$ alone (e.g., Camenen et al. 2006), we require an estimate of $k_{s}$, the equivalent Nikuradse sand grain roughness. Using the recently proposed improved curve fit to a variety of experimental data provided by Camenen et al. (2006), and our previously determined $u_{*}, k_{s} / D_{50}$ is estimated as 12 , consistent with Bennett et al. (1998), who find $8.7 \leq k_{s} / D_{50} \leq 17.4$ for spherical glass particles with similar median diameter, and on the order of the sheet thickness, consistent with Wilson (1987). Following Ligrani and Moffat (1986), we fit the log-law

$\frac{\bar{u}}{u_{*}}=\frac{1}{\kappa} \log \frac{z}{k_{s}}+B$

where $B=8.5$ is recommended given $\operatorname{Re}_{k_{s}}=k_{s} u_{*} / v=$ 130 , indicative of fully rough flow. The resultant fit, shown in Fig. 22, suggests, however, that $B$ is reduced in sheet flow as $B=6.5$ leads to a better fit. Given the uncertainty of the exact position of $z=0$ in our mobile bed experiment, critical for the assessment of $B$, this finding needs to be further assessed. The curve fit in Fig. 22 yields $\kappa=0.25$, well below the clear water accepted value of 0.41 . This is consistent again with the measurements of Bennett et al. (1998), who find $0.28 \leq \kappa \leq 0.34$, and Vanoni and Brooks (1957), who find $0.219 \leq \kappa \leq 0.299$, for similar conditions (plane bed of loose sand at sheet flow).

\section{Conclusions}

In this article, we present the design, calibration, and testing of a borescopic quantitative imaging profiler

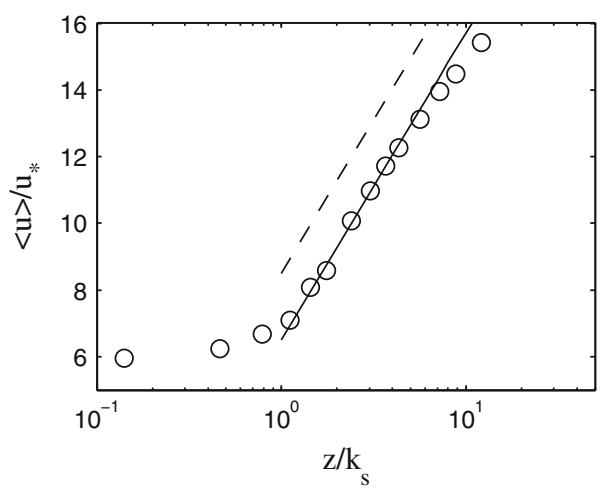

Fig. 22 Log-law normalized velocity profile: ADV circle, Eq. 6 solid line with $\kappa=0.25$ and $B=6.5$, Eq. 6 dashed line with $\kappa=0.25$ and $B=8.5$

(BQuIP) system suitable for measurements in high sediment concentration flows. While the 2-CCM approach to making sediment-fluid velocity measurements in this same regime offered an important first step in the measurement of velocity in the presence of high sediment concentrations, BQuIP, with more degrees of freedom in terms of controlling the temporal and spatial regions being correlated, is more robust. Importantly, BQuIP is sufficiently robust to be capable of making instantaneous sediment velocity measurements at high frequency. We note that we chose to work at $10 \mathrm{~Hz}$ for the present work, as we were interested in statistics, but an appropriate choice of cameras would allow velocity measurements at temporal rates greater than $1 \mathrm{kHz}$. Figures 17 and 18 demonstrate the power of the instantaneous data to reveal details of the temporal flow structure.

The presented acoustic velocity measurements demonstrate the ability to combine the two techniques and measure the entire sediment velocity profile from the immobile bed to just below the free surface. Figures 15 and 21 show the mean and stress profiles over this range, revealing a continuous and consistent velocity at the juncture of the two techniques while the stress determined by the BQuIP is perhaps a factor of two large, potentially due to motions in the $\xi$ direction. We note that we have demonstrated the importance of using an appropriate acoustic scatterer density in determining the stress, as the ADV-measured turbulent stress was only of the appropriate size when the sediment particle density was used.

Future development will focus on an instantaneous calibration of the BQuIP as opposed to a median, which should handle the motions in the $\xi$ direction. Additionally, Figs. 9 and 12 suggest the BQuIP can be calibrated for the determination of sediment concentration, which will ultimately allow the coupled measurement of sediment concentration and velocity and hence the mass flux. 
Acknowledgments The authors thank John D. Powers, Yong Sung Park, and P.J. Rusello for their help with the details of the design, construction, and testing of the BQuIP. Prof. Cowen gratefully acknowledges the John Simon Guggenheim Memorial Foundation and the Spanish Ministerio de Educación y Ciencia for supporting his time to develop the analysis approach described in this manuscript. The authors wish to thank the Office of Naval Research (N00014-051-0223) and the National Science Foundation (OCE-0452862) for supporting the hardware development described herein. Mr. Dudley gratefully acknowledges the fellowship support of the Cornell Science Inquiry Partnership (funded by the National Science Foundation DGE-0231913).

Open Access This article is distributed under the terms of the Creative Commons Attribution Noncommercial License which permits any noncommercial use, distribution, and reproduction in any medium, provided the original author(s) and source are credited.

\section{References}

Bagnold RA (1966) An approach to the sediment transport problem from general physics. Professional Paper 422-I, U.S. Geological Survey

Bennett SJ, Bridge JS, Best JL (1998) Fluid and sediment dynamics of upper stage plane beds. J Geophys Res 103(C1):1239-1274

Camenen B, Bayram A, Larson M (2006) Equivalent roughness height for plane bed under steady flow. J Hydraul Eng 132(11): 1146-1158

Cowen EA, Monismith SG (1997) A hybrid digital particle tracking velocimetry technique. Exp Fluids 33:199-211

Cowen EA, Chang KA, Liao Q (2001) A single-camera coupled PTVLIF technique. Exp Fluids 31:63-73

Dohmen-Janssen CM, Hanes DM (2002) Sheet flow dynamics under monochromatic nonbreaking waves. J Geophys Res 107(C10): 3149
Dohmen-Janssen CM, Hanes DM (2005) Sheet flow and suspended sediment due to wave groups in a large wave flume. Cont Shelf Res 25:333-247

Dudley RD (2007) A boroscopic quantitative imaging technique for sheet flow measurements. Master's thesis, Cornell University

Efron B, Tibshirani R (1993) An Introduction to the Bootstrap. Chapman \& Hall, New York

Gui LC, Merzkirch W (1996) A method of tracking ensembles of particle images. Exp Fluids 21:465-468

Gui LC, Merzkirch W (2000) A comparative study of the MQD method and several correlation-based PIV evaluation algorithms. Exp Fluids 28:36-44

Lara JL, Cowen EA, Sou IM (2002) A depth-of-field limited particle image velocimetry technique applied to oscillatory boundary layer flow over a porous bed. Exp Fluids 33(1):47-53

Liao Q, Cowen E (2005) An efficient anti-aliasing spectral continuous window shifting technique for PIV. Exp Fluids 38:197-208

Ligrani PM, Moffat RJ (1986) Structure of transitionally rough and fully rough turbulent boundary layers. J Fluid Mech 162:69-98

McLean SR, Ribberink JS, Dohmen-Janssen CM, Hassan WN (2001) Sand transport in oscillatory sheet flow with mean current. J Waterway Port Coastal Ocean Eng 127(3):141-151

Poelma C, Westerweel J, Ooms G (2006) Turbulence statistics from optical whole-field measurements in particle-laden turbulence. Exp Fluids 40:347-363

Ribberink JS, Al-Salem AA (1995) Sheet flow and suspension of sand in oscillatory boundary-layers. Coast Eng 25:205-225

Sumer BM, Kozakiewicz A, Fredsøe J, Deigaard R (1996) Velocity and concentration profiles in sheet-flow layer of movable bed. J Hydr Engrg 122:549-558

Vanoni VA, Brooks NH (1957) An approach to the sediment transport problem from general physics. Report No. E-68, California Institute of Technology

Westerweel J (1993) Digital particle image velocimetry - theory and application. PhD thesis, Delft University Press, Delft

Wilson KC (1987) Analysis of bed-load motion at high shear stress. J Hydraul Eng 113(1):97-103 